\title{
COMPORTAMIENTO DE LOSAS APOYADAS EN SUELO UTILIZANDO CONCRETO REFORZADO CON FIBRAS METÁLICAS
}

\begin{abstract}
Resumen
En este documento se analiza el comportamiento del concreto reforzado con fibras metálicas (SFRC) y se presentan los resultados de diferentes metodologías de diseño para losas apoyadas en el suelo. Específicamente, se estudiaron las metodologías de los códigos de diseño ACI 360R-06 (ACI, 2006) y TR34 (The Concrete Society, 2003) con el fin de reconocer los beneficios que conlleva la utilización del SFRC y poder obtener una mayor eficiencia en su uso.

La investigación experimental desarrollada se basó en obtener el parámetro $\mathrm{R}_{\mathrm{e}, 3}$, a partir de una resistencia de concreto y un tipo de fibra específicos con el objetivo de comprobar el comportamiento mecánico del SFRC y compararlo contra los datos de las especificaciones técnicas utilizadas.
\end{abstract}

Palabras claves: fibras metálicas, losas en suelo, naves industriales, Comité ACI 360, juntas de control, concreto reforzado con fibras.

\begin{abstract}
This article analyzes the behavior of steel fiber reinforced concrete (SFRC) and presents the results of different design methodologies for slabs supported on the ground. Specifically, the methods of ACI 360R-06 (ACI, 2006) and TR34 (The Concrete Society, 2003) design codes were studied, in order to acknowledge the benefits involved with the use of SFRC and get a more efficient use of this material.

The experimental investigation was based on obtaining the $\mathrm{R}_{\mathrm{e}, 3}$ parameter from a specific concrete strength and a specific type of fiber in order to prove the mechanical behavior of SFRC and compare it with data from the technical specifications.
\end{abstract}

Keywords: steel fibers, floor slabs, warehouses, ACI Committee 360, control joints, fiber reinforced concrete.

Recibido: 24 de agosto del 2009 • Aprobado: 11 de febrero del 2010

\section{INTRODUCCIÓN}

El concreto reforzado con fibras metálicas (SFRC) es un material compuesto; hecho con cemento hidráulico, agregados fino y grueso y una dispersión aleatoria de fibras pequeñas, las cuales le brindan características fisicomecánicas distintas a los elementos de concreto normal.

El comportamiento mecánico de elementos de concreto reforzado con fibra metálica (SFRC) es significativamente distinto al de elementos de concreto convencional; aspectos como el módulo de ruptura, el agrietamiento por temperatura, el tamaño máximo de grieta ante cargas, el mecanismo de colapso y el comportamiento esfuerzo-deformación, entre otros, son algunos de los parámetros que presentan diferencias significativas. El uso de fibras metálicas como refuerzo en el concreto conlleva ventajas de resistencia y durabilidad, tales como aumento en la ductilidad del elemento, mejora en la resistencia a cortante y flexión, mayor capacidad para absorber energía y disminución del tamaño y número de grietas en la superficie.

Por ello se requiere de una metodología de diseño para elementos de SFRC que reconozca 
las ventajas que se obtienen de conocer el comportamiento mecánico específico de este material, y que brinde una solución integral al diseño de este y sus aplicaciones en el ámbito comercial. Se reconoce que la mayor aplicación del concreto reforzado con fibras como elemento estructural se presenta en la construcción de contrapisos para uso industrial, esto debido al gran ahorro que se obtiene en el plazo de construcción y en los costos de mano de obra de este tipo de estructuras, pues se logran colar paños que cubren áreas hasta cinco veces mayores en comparación con los de concreto convencional y además, se obtienen mejoras en características de resistencia y desempeño durante el periodo de vida útil del proyecto.

El estudio del concreto reforzado con fibras metálicas (SFRC) ha sido desarrollado por distintos entes internacionales, entre los cuales se destaca el American Concrete Institute (ACI por sus siglas en inglés), especialmente el Comité 544, del cual existen distintas investigaciones en cuanto a propiedades de resistencia, deformación, condiciones de servicio y durabilidad de concreto reforzado con fibras, tanto metálicas como sintéticas (ACI, 1988; 1996). Además, se cuenta con publicaciones de la Sociedad del Concreto (The Concrete Society, 2003; 2007) y de la Unión Internacional de Laboratorios y Expertos en Materiales de Construcción, Sistemas y Estructuras (RILEM, 2000), entes europeos que también han desarrollado numerosas investigaciones en cuanto a este tema y se han encargado de tratar de unificar la normativa de diseño de SFRC bajo un mismo concepto.

En lo referente al SFRC, existe información limitada sobre metodologías de diseño. Koss (2001) realizó una investigación donde se presenta un estudio comparativo de la influencia de fibras de propileno en el comportamiento del concreto y se enfoca en realizar un estudio de las propiedades del concreto reforzado con estas fibras, utilizando un concreto con resistencia $\mathrm{f}^{\prime} \mathrm{c}=21 \mathrm{MPa}$ y $\sin$ especificar el tipo de fibra de propileno por utilizar. Avendaño (2005) desarrolló una investigación que consistió en determinar los parámetros de diseño de mezcla para concreto lanzado con fibra (shotcrete), mediante un análisis de resistencia a compresión simple y comportamiento carga-deformación. El trabajo se enfocó al estudio ante esfuerzos de flexión del concreto lanzado con fibras como material, utilizado en el sostenimiento y revestimiento del túnel del Proyecto Hidroeléctrico Cariblanco. El análisis se realizó usando dos valores de resistencia a la compresión simple de concreto (f' $\mathrm{c}=21 \mathrm{MPa}$ y f' $\mathrm{c}=28 \mathrm{MPa}$ ) y cinco tipos diferentes de fibras de refuerzo. El propósito del estudio consistió en establecer cuáles son las fibras adecuadas para la posible sustitución del acero de refuerzo convencional (malla electrosoldada) por fibras como material único de refuerzo para concreto lanzado, en el revestimiento de túneles en tierra. Por último, la investigación realizada por Álvarez (2009) se enfoca en analizar la factibilidad económica de sustituir un diseño convencional (utilizando varillas de acero) por un diseño con fibra que posea características semejantes: misma solicitud de cargas y mismas condiciones de construcción (resistencia de concreto, calidad de la base y suelo soportante, espaciamiento de juntas, mecanismos de transferencia en juntas, entre otros).

En este caso se desarrolló una investigación experimental para la comprobación y el análisis del comportamiento a flexión de elementos de SFRC, y se realizó una investigación bibliográfica para determinar las ventajas y desventajas del uso de las distintas metodologías de diseño de losas de contrapiso para uso industrial (dado que esta es la aplicación más factible para el SFRC) con el objetivo de proveer una guía de diseño segura y funcional que pueda ser utilizada por distintos profesionales y de esta manera, contribuir con la implantación de nuevas tecnologías en el mercado para mejorar la productividad y la competitividad en la construcción de este tipo de estructuras.

La investigación desarrollada procuró analizar las diferentes metodologías que existen para el diseño de elementos de concreto reforzado con fibras metálicas aplicado a losas de contrapiso para uso industrial, con el objetivo de poder establecer, mediante investigación bibliográfica y experimental, un mejor entendimiento del comportamiento de este material para mejorar la eficiencia en el uso del SFRC y lograr una reducción de costos, tanto de implementación como de construcción de estos elementos. 


\section{METODOLOGÍA DE LA INVESTIGACIÓN}

El propósito de esta investigación fue desarrollar una guía de diseño de elementos de SFRC aplicado a contrapisos de uso industrial, debido a las ventajas que se obtienen en la construcción y operación de estas estructuras por el uso de este material. Para ello, el trabajo se dividió en dos fases: una investigación bibliográfica que determinó la metodología de diseño y una investigación experimental para corroborar los conceptos inherentes al uso del SFRC y a su vez, comprobar los parámetros de diseño existentes en diferentes especificaciones técnicas de fibras metálicas.

En el caso de la investigación bibliográfica, se procedió a realizar un análisis de los códigos de diseño existentes para el diseño de contrapisos, específicamente la guía de diseño ACI 360R-06 (ACI, 2006) y los códigos TR34 (The Concrete Society, 2003) y TR63 CCIP-017 (The Concrete Society, 2007), normas europeas para diseño de losas apoyadas en suelo y para el uso de SFRC, respectivamente. Estos códigos se basan en la teoría de Westergaard y se refieren a una metodología que se expone a través de los métodos de la Portland Cement Association (1995), Wire Reinforced Institute y el Corp of Engineers (Boyd y Anderson, 1996), entre otros. El propósito de esta sección fue unificar los conceptos inherentes a la mecánica de agrietamiento del SFRC, así como las ecuaciones referentes a la capacidad de carga que poseen las losas de SFRC apoyadas en suelo.

El diseño de losas apoyadas en suelo utilizando SFRC se basa en la teoría de líneas de fluencia de Meyerhoff (enfoque plástico), en donde la adición de fibras al concreto le permite al elemento redistribuir los esfuerzos de tensión en su sección y por ende se considera una capacidad de carga residual posterior al agrietamiento. Esto modifica los supuestos definidos en la teoría de diseño convencional (teoría elástica según Westergaard) y hace obsoletos los métodos convencionales para diseño de contrapisos.

La capacidad residual del SFRC se reconoce en las ecuaciones de diseño mediante la utilización del parámetro $R_{e, 3}$, el cual es determinado mediante ensayos experimentales en vigas, de acuerdo con la norma JCI-SF4 (Japan Concrete Institute, 1984) o la norma BS EN 14651 (BSI, 2005), y es señalado por los fabricantes de este material en las distintas especificaciones técnicas para diseño. Este valor se relaciona directamente con la resistencia del concreto y la dosificación de fibras utilizada, por lo que el diseño de este tipo de elementos presenta como resultado la dosificación necesaria para alcanzar una capacidad residual requerida.

La investigación experimental desarrollada se basó en determinar este parámetro utilizando una resistencia de concreto específica (f'c $=28 \mathrm{MPa})$ con tamaño máximo nominal de agregado de $19 \mathrm{~mm}$ y un tipo de fibra (Wirand ${ }^{\circledR} \mathrm{FF} 1$ ) en especímenes tipo viga, con el objetivo de comprobar el comportamiento mecánico de estos elementos y determinar si los datos de las especificaciones técnicas tienen validez.

Para la investigación experimental se utilizó la metodología del ensayo ASTM C 1018 (ASTM, 1997) para la confección de especímenes, el desarrollo del ensayo experimental y la recopilación de datos; sin embargo, el cálculo de los valores de referencia se realizó con base en la metodología del JCI-SF4 (Japan Concrete Institute, 1984), ya que esta es la utilizada por las metodologías de diseño de SFRC citadas en ACI 360R-06 (ACI, 2006), TR 34 (The Concrete Society, 2003) y TR63 CCIP-017 (The Concrete Society, 2007). Los ensayos se realizaron en vigas moldeadas de $15 \mathrm{~cm} \mathrm{x} 15 \mathrm{~cm}$ x $55 \mathrm{~cm}$ y falladas a flexión de acuerdo con la configuración de las Figuras 1 y 2.

Como se puede observar en la Figura 1, se utilizó un LVDT (linear voltage differential transducer) para la medición de la deflexión central del elemento, este dispositivo tiene una precisión de $10^{-6} \mathrm{~mm}$. La carga se aplicó mediante un gato hidráulico (Figura 2) el cual se controla por deformación, aplicando deformaciones a razón de 0,075 mm/min, según ASTM C1018 (ASTM, 1997).

A través de estos ensayos se obtuvieron la tenacidad y la carga de agrietamiento de la matriz de concreto, utilizados para el cálculo de la resistencia residual o equivalente del SFRC, que a su vez se calcula mediante la energía absorbida hasta una deflexión de 3,0 $\mathrm{mm}$. La tenacidad $\mathrm{T}_{\mathrm{JCI}}$, corresponde a la energía absorbida por la 
viga y se calcula midiendo el área bajo la curva del diagrama carga-desplazamiento hasta una deflexión correspondiente al claro entre apoyos dividido entre $150, \delta_{150}$. Se utiliza la ecuación (1)

$$
f_{c t f l, e q 3}=\frac{T_{J C I} \cdot L}{\delta_{150} \cdot b \cdot h^{2}}
$$

Donde:

$L \quad$ es la longitud del claro entre apoyos del ensayo (mm)

$B \quad$ es el ancho de viga de ensayo ( $\mathrm{mm}$ )

$H \quad$ es el peralte de viga de ensayo $(\mathrm{mm})$

$\mathrm{T}_{\mathrm{JCI}} \quad$ es el área bajo la curva $\mathrm{P}-\delta$ hasta una deflexión de 3,0 mm

$\delta_{150} \quad$ es la deflexión correspondiente al claro entre apoyos dividido entre 150 (L/150)

Con respecto a las ecuaciones de diseño, el valor de $\mathrm{R}_{\mathrm{e}, 3}$, obtenido en este ensayo está dado por la ecuación (2).

$$
\mathrm{R}_{\mathrm{e}, 3}=\frac{\mathrm{f}_{\mathrm{ctfl}, \mathrm{eq} 3}}{\mathrm{f}_{\mathrm{ctm}, \mathrm{fl}}}
$$

donde:

$\mathrm{f}_{\mathrm{ctm}, \mathrm{fl}} \quad$ es la resistencia a flexión del concreto, que se calcula mediante la carga máxima del ensayo, $P$, antes del inicio del agrietamiento

$$
f_{c t m, f l}=\frac{P L}{b h^{2}}
$$

El diseño de mezcla de concreto se realizó para una resistencia a la compresión simple a 28 d de 28,0 MPa. Se utilizó un solo tipo de fibra (Figura 3), variando la dosificación en el concreto $(15,20,25$, y 40$) \mathrm{kg} / \mathrm{m}^{3}$ para comprobar su efecto sobre las propiedades del SFRC.

\section{RESULTADOS}

Los resultados obtenidos para el comportamiento P- $\delta$ y para el parámetro $R_{e, 3}$ presentaron una muy buena correlación con lo señalado teóricamente y con las especificaciones técnicas de este material.

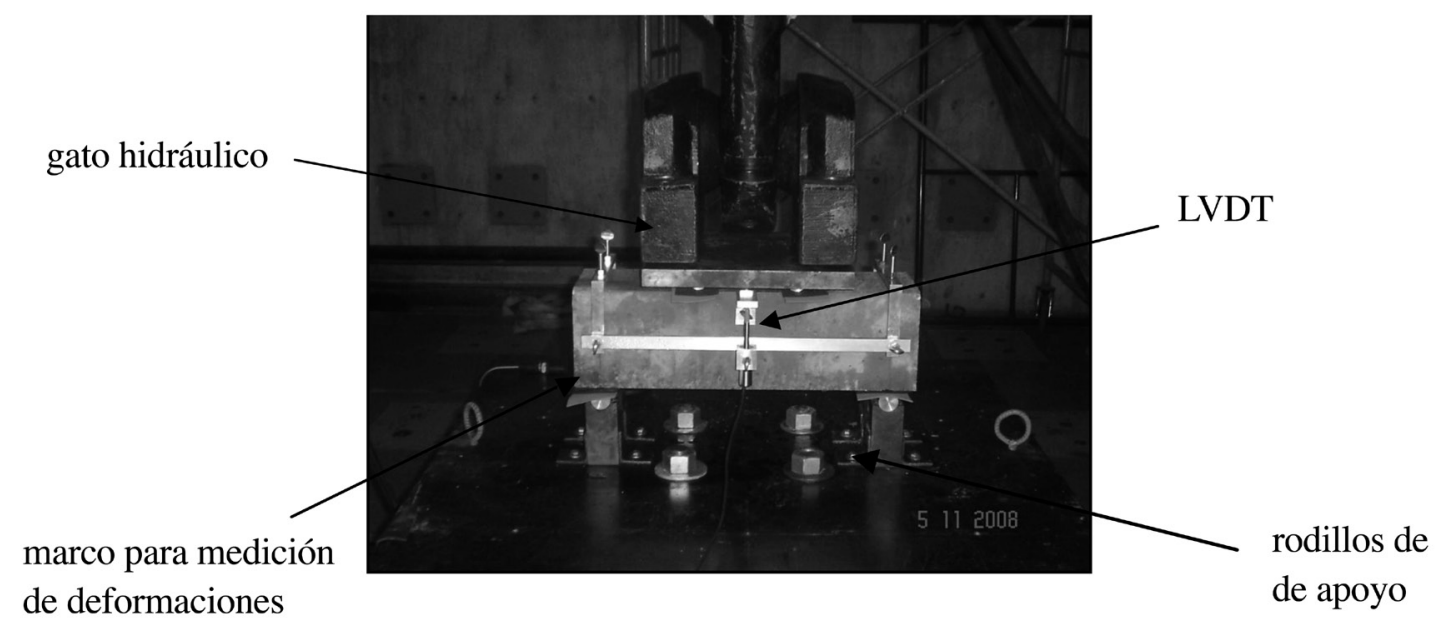

Figura 1. Montaje para el ensayo a flexión ASTM C 1018.

Fuente: (El autor). 


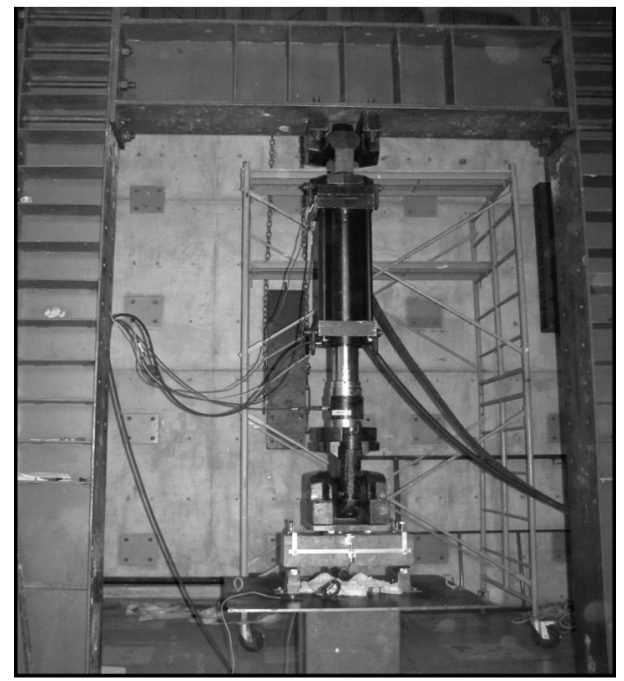

Figura 2. Equipo utilizado para el ensayo a flexión en vigas según ASTM C 1018.

Fuente: (El autor).

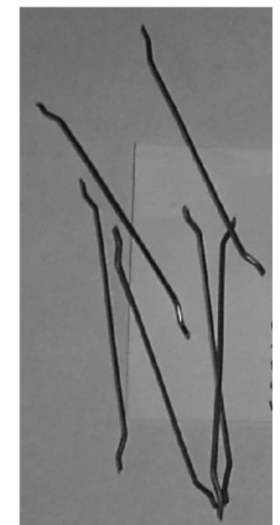

Wirand ${ }^{\circledR}$ FF1

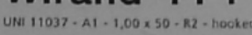

Figura 3. Fibras Wirand ${ }^{\circledR}$ FF1 utilizadas en esta investigación.

Fuente: (El autor).

Los resultados obtenidos para el comportamiento $\mathrm{P}-\delta$ de cada dosificación se muestran en las Figuras $4,5,6$ y 7 .

Con base en el análisis de estas curvas y en la metodología experimental comentada anteriormente se obtuvieron los resultados para los parámetros de diseño, los cuales se muestran en los Cuadros del 1 al 4.
Posteriormente se procedió a realizar el diseño de un contrapiso para uso industrial utilizando concreto reforzado convencional y SFRC. En este caso se pudo observar cómo el uso de fibras permitió disminuir en un 27,2 \% el espesor de concreto necesario para este contrapiso, comprobando la ventaja competitiva que supone el uso de fibras en el concreto. 


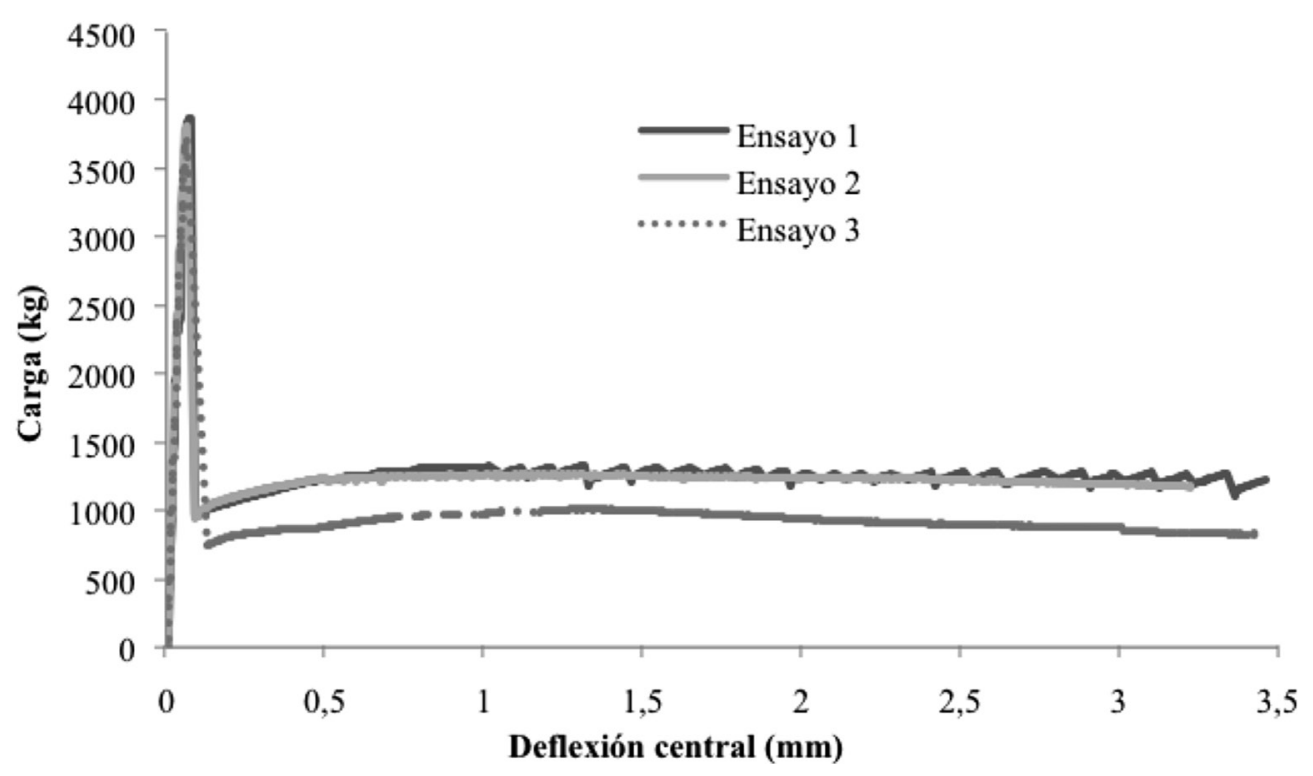

Figura 4. Curvas $P-\delta$ para los ensayos a flexión realizados para la dosificación $15 \mathrm{~kg} / \mathrm{m}^{3}$. Fuente: (El autor).

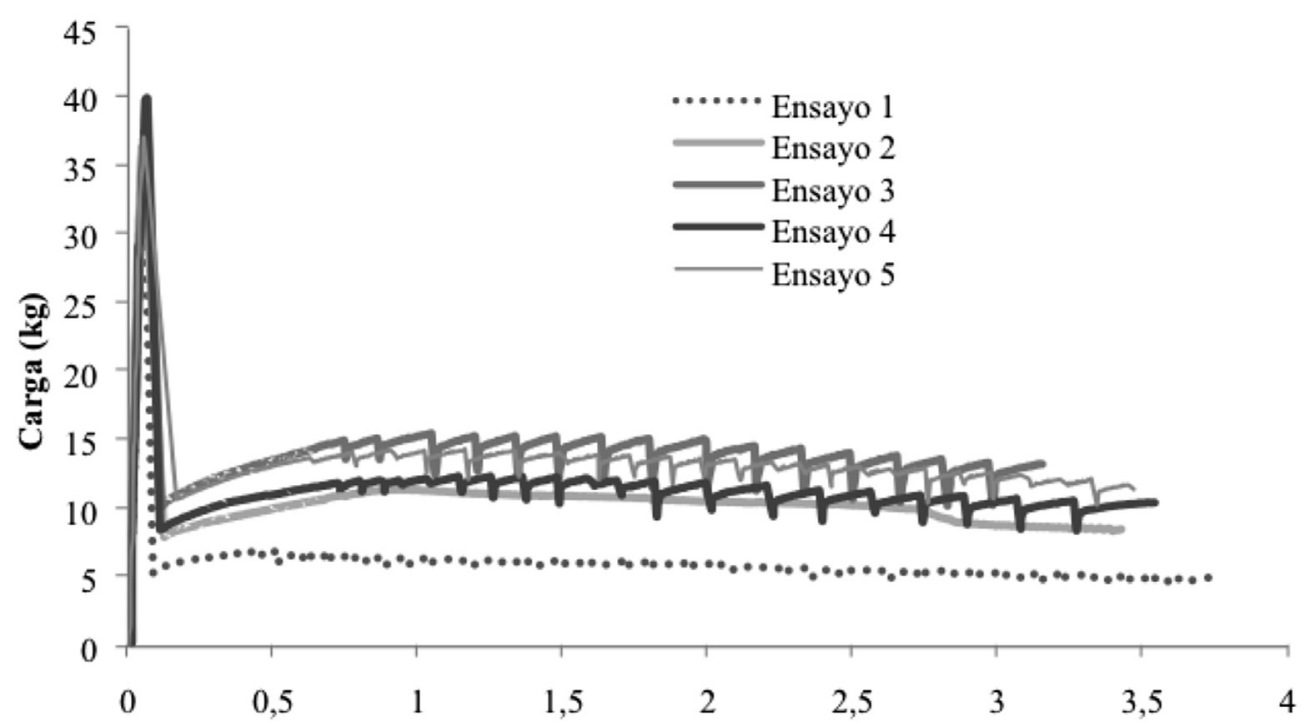

Figura 5. Curvas P- $\delta$ para los ensayos a flexión realizados para la dosificación $20 \mathrm{~kg} / \mathrm{m}^{3}$.

Fuente: (El autor). 


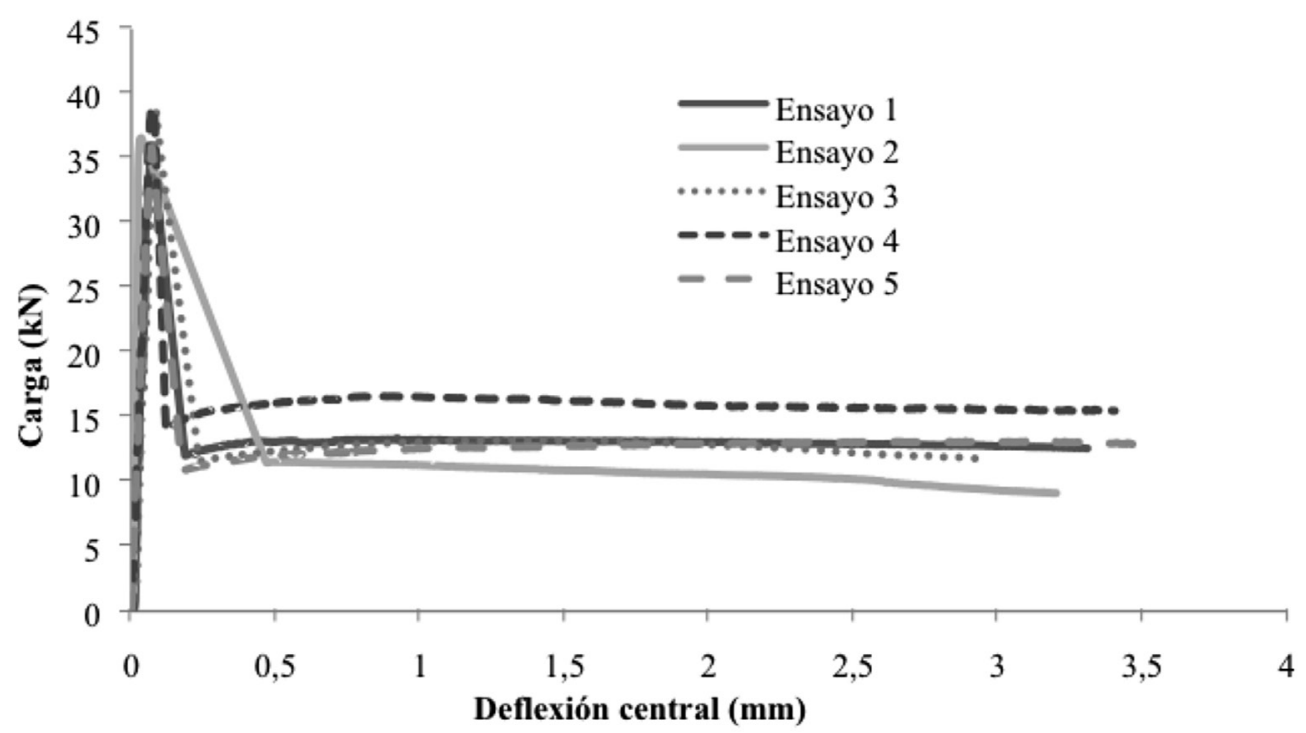

Figura 6. Curvas P- $\delta$ para los ensayos a flexión realizados para la dosificación $25 \mathrm{~kg} / \mathrm{m}^{3}$.

Fuente: (El autor).

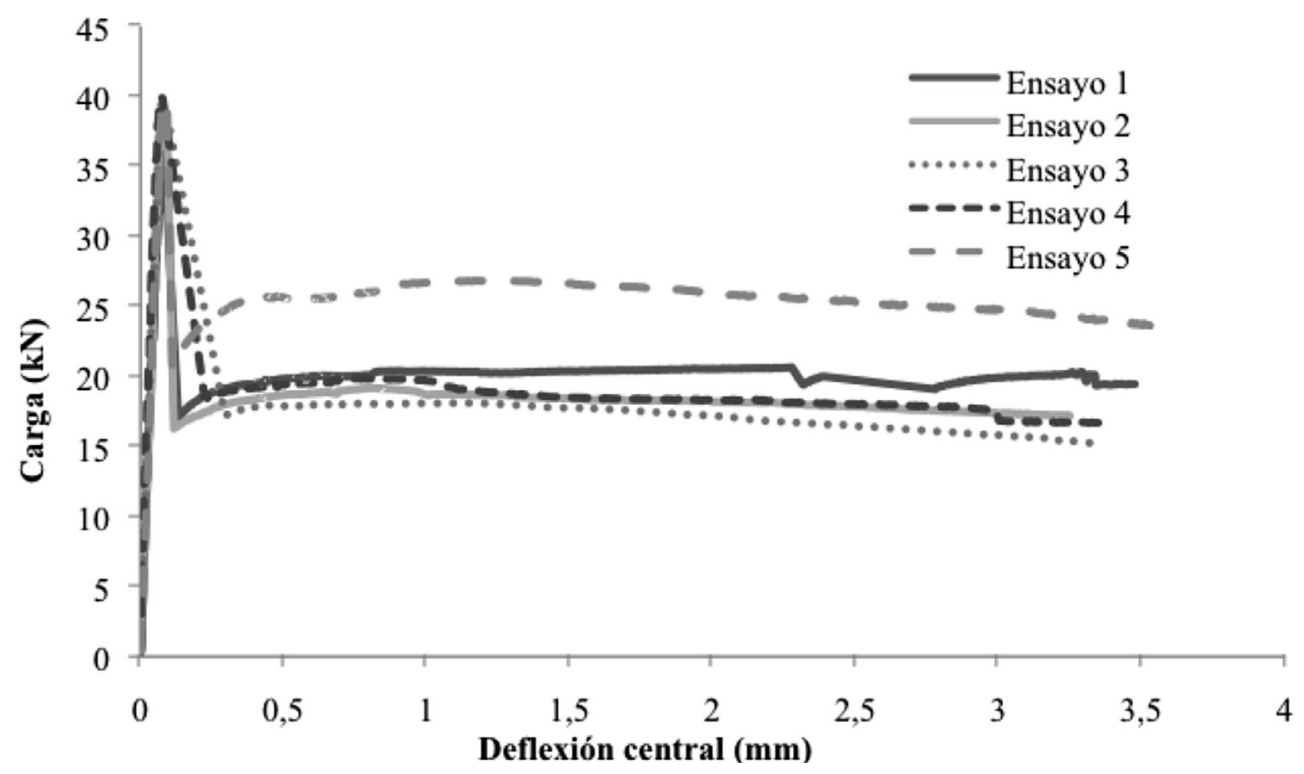

Figura 7. Curvas P- $\delta$ para los ensayos a flexión realizados para la dosificación $40 \mathrm{~kg} / \mathrm{m}^{3}$.

Fuente: (El autor). 
Cuadro 1. Resultados promedio obtenidos para el valor de resistencia última de flexión equivalente, $\mathrm{f}_{\text {ctfl,eq3. }}$.

\begin{tabular}{cccc}
\multicolumn{2}{c}{$\begin{array}{c}\text { Dosificación } \\
\left(\mathrm{kg} / \mathrm{m}^{3}\right)\end{array}$} & \multicolumn{2}{c}{$\begin{array}{c}\mathbf{f}_{\mathrm{ctfl}, \mathrm{eq} 3} \\
\left(\mathrm{~N} / \mathrm{mm}^{2}\right)\end{array}$} \\
Promedio & Límite inferior & Promedio & Límite superior \\
15 & 1,259 & 1,470 & 1,680 \\
20 & 1,373 & 1,576 & 1,780 \\
25 & 1,561 & 1,732 & 1,902 \\
40 & 2,281 & 2,643 & 3,006 \\
& \multicolumn{2}{c}{ Fuente: (El autor). }
\end{tabular}

Cuadro 2. Resultados promedio obtenidos para el valor de resistencia a flexión del concreto, $\mathrm{f}_{\mathrm{ctm}, \mathrm{fl}}$.

$\begin{array}{cc}\text { Dosificación } & \begin{array}{c}\text { Resistencia a la flexión equivalente } \\ \text { última } \mathbf{f}_{\mathrm{ctm}, \mathrm{fl}}\end{array} \\ \left(\mathrm{kg} / \mathrm{m}^{3}\right) & \left(\mathrm{N} / \mathrm{mm}^{2}\right) \\ 15 & 4,731 \\ 20 & 4,767 \\ 25 & 4,676 \\ 40 & 4,815\end{array}$

Fuente: (El autor).

Cuadro 3. Resultados promedio del índice de flexión equivalente (o residual) en función de la dosificación en el concreto.

Dosificación

$\left(\mathrm{kg} / \mathrm{m}^{3}\right)$

15

20

25

40
Índice de flexión equivalente

$\mathrm{R}_{\mathrm{e}, 3}$

31,0

33,1

37,4

53,7

Fuente: (El autor). 

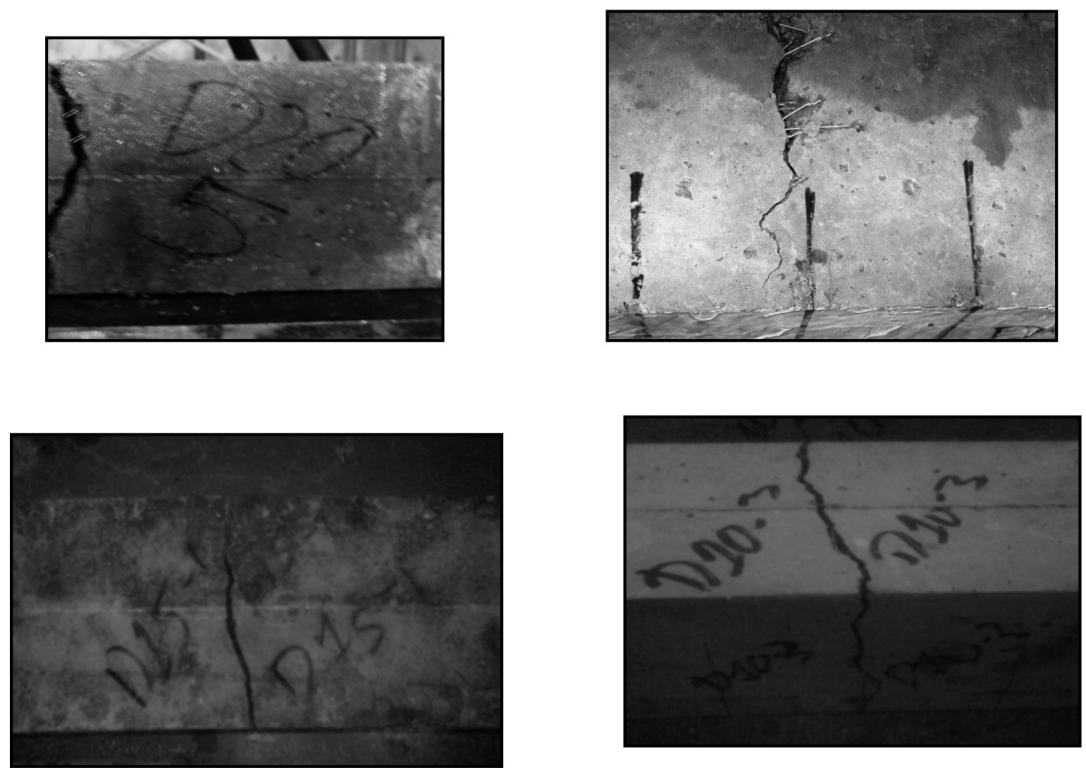

Figura 8. Ejemplos de elementos de ensayos a flexión.

Fuente: (El autor).

Cuadro 4. Comparación de resultados para el diseño del caso de estudio según las metodologías de diseño analizadas.

Método Westergaard

Resistencia a la

compresión del concreto

(MPa)

Resistencia a la flexión del concreto (MPa)

Módulo de reacción del suelo $\left(\mathrm{kg} / \mathrm{cm}^{3}\right)$

Espaciamiento entre juntas (m)

Espesor de concreto (m)

Refuerzo convencional

Superior: \#4@30 cm

Inferior: \#5 @ 20 cm

Inferior: \# 5 @ 25 cm

Inferior: \#5@25 cm

\section{Método PCA Método para SFRC}

27,5

1,57

3,80

21,8

9,0

0,16

Dosificación de fibras 


\section{ANÁLISIS DE RESULTADOS}

Como se puede observar en las Figuras 4, 5, 6 y 7, el comportamiento de elementos de concreto reforzado con este tipo de fibra corresponde a un comportamiento suavizado (ablandamiento de tensión) (strain softening), en el cual es claro que con aumentos en la dosificación de fibra se aumenta la capacidad de resistir carga, hecho que se muestra en los valores del índice de flexión residual $\left(R_{e, 3}\right)$ y resistencia última a flexión equivalente $\left(\mathrm{f}_{\text {ctfl,eq3 }}\right)$. En investigaciones desarrolladas a nivel mundial (Barros, Cunha, Ribeiro y Antunes, 2004; Fernández, 2004; Meda y Plizarri, 2004) con fibras de una relación $1 / \mathrm{d}=60$ se ha obtenido este tipo de comportamiento hasta dosificaciones de $40 \mathrm{~kg}$, las cuales se convierten en un límite del comportamiento suavizado y a partir del cual se observa el comportamiento endurecido.

A su vez, estas figuras permiten apreciar los miniciclos de carga y descarga que ocurren durante el desarrollo de estos ensayos, lo cual se atribuye a la mecánica propia del concreto fibroreforzado, ya que estas disminuciones de carga son producto de la extracción de fibras de la matriz del concreto, hecho que produce una reducción de la carga resistida y un aumento en la deformación, pero que es rápidamente compensado por la redistribución interna de esfuerzos que se produce en la sección y que permite que la carga vuelva a restituirse inmediatamente. Las dosificaciones de $25 \mathrm{~kg} / \mathrm{m}^{3}$ y $40 \mathrm{~kg} / \mathrm{m}^{3}$ presentaron este fenómeno de forma menos acentuada en comparación con las otras dos dosificaciones.

Con respecto a los datos obtenidos en los Cuadros 1, 2 y 3 para los parámetros de diseño, estos se comparan con datos de especificaciones técnicas existentes en el mercado (Bekaert, 2009; Maccaferri, 2007), de los cuales se considera la fibra Dramix 45/50 que posee una relación 1/ $\mathrm{d}=48$ y que por sus características es similar al material utilizado en esta investigación. El valor de $R_{e, 3}$ de un concreto reforzado con este tipo de fibra y para una dosificación de $40 \mathrm{~kg} / \mathrm{m}^{3}$ es del $63 \%$, el cual comparado con el resultado de esta investigación (55\%), muestra ser un resultado similar. En estas especificaciones no se presentan valores para dosificaciones menores a $20 \mathrm{~kg} / \mathrm{m}^{3}$, pues se considera que esta cantidad solo produce beneficios para agrietamiento temprano (o por temperatura) y no como refuerzo estructural; sin embargo, se puede observar que la dosificación de $20 \mathrm{~kg} / \mathrm{m}^{3}$ de acuerdo con estas especificaciones posee un índice de resistencia residual $\left(\mathrm{R}_{\mathrm{e}, 3}\right)$ del $38 \%$, y los resultados de este trabajo muestran una $\mathrm{R}_{\mathrm{e}, 3}$ del $33 \%$. Este tipo de argumentos parecen fundamentar la recomendación de la mayoría de especificaciones de diseño, en las cuales se expone que si las fibras han de ser utilizadas como refuerzo estructural se deben dosificar en cantidades superiores a $20 \mathrm{~kg} / \mathrm{m}^{3}$ ya que el valor de $\mathrm{R}_{\mathrm{e}, 3}$ debe ser mayor al $30 \%$.

En cuanto al momento nominal calculado a partir de los resultados experimentales y las diferentes metodologías, como era de esperar, la metodología para cálculo de momento por el TR63 CCIP-017 (The Concrete Society, 2007) obtuvo los resultados más conservadores, debido a que se aplica un factor de seguridad tanto al bloque en compresión como al bloque en tensión, donde los valores de resistencia se dividen entre 1,5. Es importante resaltar que los momentos nominales o capacidades de los elementos de SFRC se presentan en función de una deformación máxima permisible (o abertura de grieta máxima) que puede sufrir un elemento al ser sometido a cargas de flexión. En el caso de las recomendaciones y normas de diseño, se establece que esta deformación corresponde a $3,0 \mathrm{~mm}$, ya que ante deformaciones mayores, el elemento aunque pueda ser capaz de resistir cargas (tal como se observó en los resultados de los ensayos), se vuelve poco seguro, inestable, y poco funcional; por lo que este límite se presenta como un estado de falla, tal y como lo es el agrietamiento para el concreto normal y la deformación unitaria en compresión del concreto ( $\varepsilon=0,003)$ y en tensión del acero $\left(\varepsilon_{\mathrm{b}}=0,002\right)$ en concreto reforzado convencional. Es por ello que los resultados experimentales de carga a los 3,0 mm se comparan con la carga teórica necesaria para alcanzar el momento nominal de cada metodología, obtenida mediante análisis elástico de equilibrio de fuerzas. 
Dentro del análisis metodológico se observa que todas las metodologías reconocen la presencia de las fibras mediante la utilización de la resistencia de flexión equivalente o el índice de flexión equivalente $\mathrm{R}_{\mathrm{e}, 3}$, y la resistencia de primer agrietamiento del concreto. En la comparación directa de las metodologías se observa que las correspondientes a los manuales son menos conservadoras, ya que los valores teóricos de carga máxima se aproximan a los obtenidos experimentalmente con diferencias de entre $25 \%$ a $35 \%$ mayores; en comparación con las obtenidas para el TR34 (The Concrete Society, 2003) con diferencias del $85 \%$ al $100 \%$ y el TR63 CCIP-017 (The Concrete Society, 2007) donde la carga máxima teórica es 1,5 ó 2,0 veces menor que la experimental. Este resultado se debe a que en la metodología del TR34 (The Concrete Society, 2003) se multiplica la carga por un factor de seguridad del material, hecho que no se aplica en la metodología Wirand o Dramix ya que estas exponen que se están aprovechando las características últimas del material observadas en ensayos de laboratorio y por ende, para el cálculo de momento no se debe aplicar ningún factor de reducción.

Las metodologías del TR34 (The Concrete Society, 2003) y el manual Wirand hacen uso de la teoría de líneas de fluencia (investigaciones de Meyerhoff y Losberg) para el cálculo de una carga última resistente de la losa y en función de su posición sobre la losa se obtienen ecuaciones para cada caso. Esta carga deberá ser mayor que la resultante de fuerzas de la carga aplicada, multiplicada por un factor de amplificación.

Con base en el análisis de los resultados experimentales y la investigación bibliográfica, se concluye que la metodología de cálculo del TR34 (The Concrete Society, 2003) es eficiente, funcional y segura, por lo que el cálculo del caso de estudio se procedió a realizar bajo estos conceptos.

En conclusión, los resultados de los ensayos experimentales demuestran la capacidad que posee el SFRC para mantener cierta solicitud de carga y deformarse ante esta, comprobando la capacidad de la sección para redistribuir esfuerzos y por ende, presentar un comportamiento de ductilidad moderada. Este comportamiento implica una modificación en los conceptos básicos para el diseño de elementos de concreto, en el tanto se reconoce la existencia de cierta capacidad a tensión en el concreto y así, tener una mayor capacidad de momento flector. Como se analizó, las metodologías existentes para el diseño de este material requieren de parámetros obtenidos a nivel experimental; en la investigación desarrollada se encontraron especificaciones que indican estos valores, los cuales fueron comprobados mediante los ensayos realizados, en el tanto los valores obtenidos muestran una buena aproximación con los valores especificados.

A su vez se logró comprobar que las ecuaciones de diseño para capacidad de momento proveen de una buena estimación para la carga máxima, obteniendo resultados de carga última teóricas menores a las experimentales, que demuestran que estas ecuaciones se encuentran del lado de la seguridad y proporcionan un buen uso de las capacidades últimas del material, al estar basadas en parámetros de laboratorio que se obtienen de llevar el material hasta estados de falla o de límite funcional.

\section{CONCLUSIONES}

1. Se comprobó que el concreto reforzado con fibras metálicas SFRC presenta una alta capacidad de absorber energía (tenacidad), lo cual demuestra la distribución interna de esfuerzos que se presenta en la sección, producto de la mecánica matriz-fibra existente.

2. Se comprobó la dependencia existente entre la capacidad residual a flexión del SFRC y la dosificación de fibras utilizada en su elaboración, ya que se observó que para los resultados experimentales, la capacidad de carga residual aumentó según aumentó la dosificación.

3. Se logró comprobar que los valores del índice residual de resistencia a flexión, $\mathrm{R}_{\mathrm{e}, 3}$, definidos en diferentes especificaciones de fibras pueden ser utilizados en el diseño, ya que la investigación experimental mostró una buena correlación con estas indicaciones.

4. Dentro del procedimiento experimental no se logró observar un comportamiento tipo "plástico absoluto" o de "strain hardening" 
para la dosificación de $40 \mathrm{~kg} / \mathrm{m} 3$, hecho que se describe como típico para dosificaciones iguales o mayores a esta, pero que para el tipo de fibra utilizado no se logró comprobar.

5. La metodología expuesta por el TR34 (The Concrete Society, 2003) presenta los resultados más precisos y confiables para la determinación de la capacidad de carga máxima que puede soportar la losa de SFRC.

6. Las metodologías de la PCA, WRI y COE deben ser utilizadas como metodologías para un diseño preliminar; ya que estas poseen muchas restricciones en cuanto a los datos de entrada y además sus nomogramas fueron confeccionados para cargas internas, por lo que las condiciones de borde y esquina no se revisan confiablemente.

7. Para el diseño convencional, la metodología de las ecuaciones de Westergaard es adecuada y funcional pues en ella sí se consideran los diferentes estados de cargas concentradas a los que se puede someter la losa y se puede determinar la solicitud de cargas por retracción y temperatura, de manera que se logra determinar con buena exactitud y confiabilidad el espesor necesario para evitar agrietamiento en la losa.

8. El uso de fibras de acero en el concreto y el comportamiento dúctil inherente a este material supone que el diseño del concreto pueda ser realizado mediante enfoques plásticos, en los cuales se logre llevar el material a estados últimos de resistencia y por ende se obtengan espesores menores que los necesarios para una losa de concreto con refuerzo convencional.

9. En el diseño de losas apoyadas en suelo, la hiperestaticidad del elemento es primordial para que la mecánica de agrietamiento del SFRC llegue a presentarse y así el diseño, mediante la metodología del TR34 (The Concrete Society, 2003) y el ACI 360R-06 (ACI, 2006) tenga validez. Esto implica que la base de apoyo sobre la cual reside la losa debe construirse mediante un estricto control de calidad con el objetivo de evitar imperfecciones en su superficie que puedan generar esfuerzos sobre la losa o modificar esta condición de apoyo.

\section{NOMENCLATURA}

SFRC: concreto reforzado con fibras metálicas, por sus siglas en inglés.

$\mathrm{f}_{c}^{\prime}$ : resistencia a compresión del concreto a los $28 \mathrm{~d}$. $\mathrm{f}_{\text {ctk, } 005}$ : resistencia a tensión mínima del concreto.

$\mathrm{f}_{\mathrm{ctm}, \mathrm{fl}}:$ resistencia a flexión experimental.

$\mathrm{f}_{\text {ctk ff: }}$ : resistencia mínima a flexión, utilizada en los códigos de diseño.

$\mathrm{f}_{\text {ctffeeq }}$ : resistencia residual a flexión del concreto reforzado con fibras.

$\mathrm{R}_{\mathrm{e}, 3}$ : índice de resistencia residual o equivalente a flexión, derivado de ensayos experimentales.

$\mathrm{T}_{\mathrm{JCI}, 3}=$ área bajo la curva $\mathrm{P}-\delta$ hasta una deflexión de $3,0 \mathrm{~mm}$.

$\delta_{150}=$ deflexión correspondiente al claro entre apoyos dividido entre 150 (L/150).

\section{REFERENCIAS BIBLIOGRÁFICAS}

ACI (American Concrete Institute). (1988). Design considerations for steel fiber reinforced concrete ACI 544.4R-88. Reported by Committee 544. Detroit, MI: American Concrete Institute.

ACI (American Concrete Institute). (1996). State-of-the-art report on fiber reinforced concrete ACI 544.1R-96. Reported by Committee 544. Detroit, MI: American Concrete Institute.

ACI (American Concrete Institute). (2006). Design of slabs on ground ACI 360R-06. Reported by Committee 360. Detroit, MI: American Concrete Institute.

ACI (American Concrete Institute). (2008). Building code requirements for reinforced concrete and commentary, ACI 318-08. Reported by Committee 318. Detroit, MI: American Concrete Institute. 
Álvarez, V. (2009). Análisis comparativo teórico y económico entre losas de concreto apoyadas sobre suelos utilizando refuerzo tradicional y fibras de acero. Tesis para optar por el grado de Licenciado en Ingeniería Civil, Universidad de Costa Rica, San José, Costa Rica.

ASTM (American Society for Testing and Materials). (1997). Standard test method for flexural performance of fiber reinforced concrete (using beam with third-point loading) ASTM C 1018. Pennsylvania: ASTM.

Avendaño, G. (2005). Análisis de la resistencia a compresión simple y flexión del concreto lanzado con fibra como material de refuerzo. Tesis para optar por el grado de Licenciado en Ingeniería Civil, Universidad de Costa Rica, San José, Costa Rica.

Barros, J. O., Cunha, V. M. C:F.: Antunes, A:O: (2004). Post-cracking behavior of steel fibre reinforced concrete. RILEM publications Materials and structures (38). France. February, 2005.

Bekaert, Ltd., (2009). Concrete reinforcement products [en línea]. Latin America. Extraído el 22 de febrero del 2009 de: http://www. bekaert.com/en/Product\%20Catalog/ Products/D/Dramix\%20for20\%concrete\%20 reinforcement.aspx?Industry

Boyd, R. \& Anderson, R. (1996). Designing floor slabs on grade: step-by-step procedures, sample solutions and commentary. Addison, IL: Hanley Wood.

BSI (British Standards Institution). (2005). Test method for metallic fiber concreteMeasuring the flexural tensile strength, limit of proportionality (LOP), and flexural tensile residual strength BS EN 14651. London: BSI.
Fernandes, V. M. (2004). Investigação experimental e numérica do comportamento à tracção de betão reforçado com fibras de aço. Tese para a obtenção do grau de Mestre. Faculdade de Engenharia da Universidade do Minho, Brasil.

Koss, I. (2001). Concreto reforzado con fibras de polipropileno. Tesis para optar por el grado de Licenciado en Ingeniería Civil, Universidad de Costa Rica, San José, Costa Rica.

Japan Concrete Institute. (1984). Method of tests for flexural strength and flexural toughness of fiber reinforced concrete, JCI-SF4. Japan Concrete Institute.

MACCAFERRI. (2007). Fibras de acero como elemento estructural para el refuerzo del concreto. Manual Técnico. Brasil: Maccaferri.

Meda, A. \& Plizzari, G. A. (2004). A new design approach for SFRC slabs on grade base don fracture mechanics. ACI Structural Journal, 101(3), 298-303.

Portland Cement Association (PCA). (1995). Concrete floors on ground. (3a ed.), Stokie, IL: The Portland Cement Association.

RILEM. (March 2000). Final recommendations of TC 162-TDF, Test and design methods for steel fiber reinforced concrete, $\sigma-\varepsilon$ design method. Materials and Structures, 36(225).

The Concrete Society. (2003). Concrete industrial ground floors - A guide to design and construction. Technical Report No. 34, (3a ed.), The Concrete Society, UK.

The Concrete Society. (2007). Guidance for the design of steel fiber-reinforced concrete. Technical Report No. 63 CCIP-017, (1a ed), The Concrete Society, UK. 


\section{SOBRE LOS AUTORES}

\section{Alejandro Navas Carro}

M. Sc. Ingeniería Civil

Profesor de la Escuela de Ingeniería Civil, de la Universidad de Costa Rica

Director del Laboratorio Nacional de Materiales y Modelos Estructurales (LANAMME) de la Universidad de Costa Rica

Apartado Postal 2060, UCR, San José Costa Rica
Teléfono: 2511-4186

Facsímil: 2511-4440

Correo electrónico: anavas@lanamne.ucr.ac.cr

\section{José Luis Rojas Juárez}

Ingeniería Civil

Coordinador de Construcción

Las Torres DCR S. A.

Correo electrónico: jose.rojas@rdsani.com 